\title{
A systematic procedure to obtain the block diagram model from the bond graph model
}

\author{
Radu Ibănescu ${ }^{1, *}$ and Mihaela Ibănescu ${ }^{2}$ \\ 1"Gheorghe Asachi” Technical University of Iași, Department of Theoretical Mechanics, \\ Bd. Dimitrie Mangeron, nr. 59A, Iași, Romania \\ 2"Gheorghe Asachi” Technical University of Iași, Department of Structural Mechanics, \\ Bd. Dimitrie Mangeron, nr. 1, Iași, Romania
}

\begin{abstract}
The bond-graph method for the analysis of systems dynamics is very widespread in engineering and, because of its simplicity and its advantages, it has been developed a lot in the last decades. The bond graph model is a diagram which describes the manner of transmitting and transforming the power in a system, starting from the source up to the final elements. One of the most preferred ways of using the bond-graph diagram is the construction of the block diagram, appropriate for an adequate soft, the most frequently used one being MATLAB-SIMULINK. The block diagram model has a wide range of application in the study of systems dynamics because of its great advantages in case of numerical simulations. The manner of obtaining the block diagram from the bond-graph diagram is left at researcher choice, because there are no clear and well-defined procedures. The work presents a systematic procedure which contains several well-defined steps that lead to an exact and unitary approach of this problem.
\end{abstract}

\section{Introduction}

The bond-graph method is one of the most frequently used methods for systems dynamics modelling and numerical simulation. It is based on the analysis of power transfer and transformation between the elements of the system [1-3]. The analysis result is expressed as a diagram that is constructed by using nine basic generic elements, no matter the types of energy are considered for the system [4-5]. This diagram represents the bond-graph model, which can be exploited in three different manners [6]. One of them consists in obtaining from such a bond-graph model a mathematical model, which contains differential equations or differential-algebraic equations [7]. A second possibility is to draw, based on the bondgraph model, a diagram, called block-diagram model, [8], that is further implemented in a dedicated software, as MATLAB-SIMULINK [9]. The construction of this block-diagram is a very simple task and it does not presume to write any equation of motion. It provides information about any variable in the system in an easy way. These advantages make the block-diagram the favourite manner of using the bond-graph model. The third possibility is to draw directly the bond-graph model in a dedicated software which can be used for this

*Corresponding author: ribanesc@yahoo.com 
purpose only, as it is 20-sim [10], while MATLAB and SIMULINK offer the possibility of resolving a lot of many other modelling and simulation problems.

\section{Block-diagram construction}

The construction of the block-diagram model, based on the bond-graph model, starts from drawing a diagram which is the so-called the diagram of signals processing. It results from the bond-graph diagram by transforming each bond in two arrows, each of them corresponding to one of the two signals (effort and flow, respectively), whose product is the power transmitted by the bond. The arrow of the signal "effort" is always oriented towards the bond-graph element located near the causal stroke and the arrow of the signal "flow" is in the opposite direction. Near each arrow the symbol "e" or "f" is written, having as subscript the number of the bond. The next step consists in drawing a preliminary blockdiagram based on the signals orientation from the diagram of signal processing. For each 1junction (J1), a node-summation pair is drawn. So, to a junction $\mathrm{J} 1$, a summation for efforts and a node for flow correspond. There is a single output effort signal getting out from the summation, all the others being input ones. The input efforts get a plus or a minus sign. These signs result from the junction power equilibrium equation, when the output effort is determined. At the node there is a single input flow signal and all the other output flows will have the same magnitude. To a 0 -junction (J0), a summation for flows and a node for efforts correspond. At the summation there is a single output flow signal and all the others are input flows. The input flows sign (plus or minus) results from the power equilibrium equation on the junction, when the output flow is determined. At the node there is a single input effort signal and all the other output efforts will have the same magnitude. The preliminary block-diagram drawing starts with the drawing of a node-summation pair of a junction, usually the first one after a source. From this point, the drawing continues according to model constructor option, the result being usually difficult to understand and to introduce in computer, because of many crossing signal lines. The present paper proposes a method which allows a clear and effective construction of the preliminary diagram. The summations and nodes are drawn on two columns. On the first column there are the summations corresponding to junction $\mathrm{J} 1$ and the nodes corresponding to junction J0. On the second column there are the nodes corresponding to junction $\mathrm{J} 1$ and the summations corresponding to junction $\mathrm{J} 0$. The summation and the node of a junction will be located on different columns, but on the same row. The elements I, C and R will be adequately substituted by integrator blocks, derivative blocks and gain blocks, which will be represented between the corresponding summations and nodes. In this manner, a unitary block-diagram results and it contains a column for flows and a column for efforts, between which integrator blocks, derivative blocks and gain blocks are considered. The elements TF are located one on each column, of efforts and flows, respectively, at the corresponding places. The preliminary block-diagram is now easily drawn in MATLAB-SIMULINK or other dedicated software.

\section{Example of block-diagram construction for a mechanical system}

The mechanical system shown in Figure 1 is considered. It contains a lever having the lengths of the arms $l_{1}$ and $l_{2}$. The masses $m_{1}$ and $m_{2}$ are provided at their ends. A sinusoidal force $F=A \sin \left(\omega_{0} t\right)$ acts at the left end. A mass $m_{3}$ is attached to the right end by using a spring, whose stiffness is $k_{1}$ and a couple spring-damper, characterized by stiffness $k_{2}$ and viscous friction coefficient $\gamma$, denoted by $g$ in the block diagram model. 


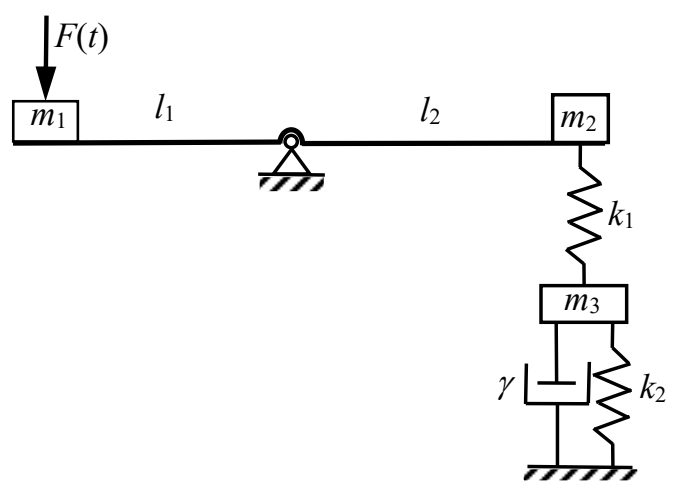

Fig. 1. The mechanical system.

The system bond-graph is pictured in Figure 2. It contains two elements I in integral causality, an element I in derivative causality and an element $\mathrm{C}$ in integral causality.

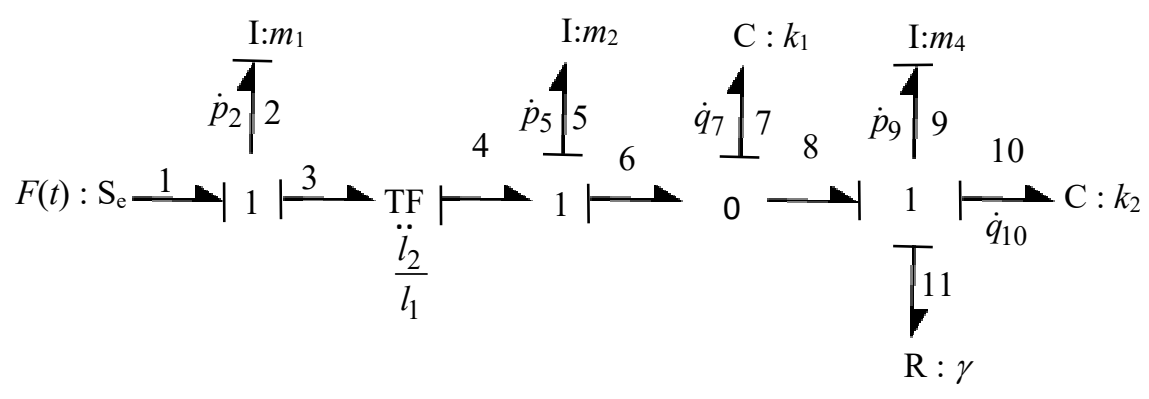

Fig. 2. The bond graph model of the mechanical system.

The signals processing diagram is presented in Figure 3.

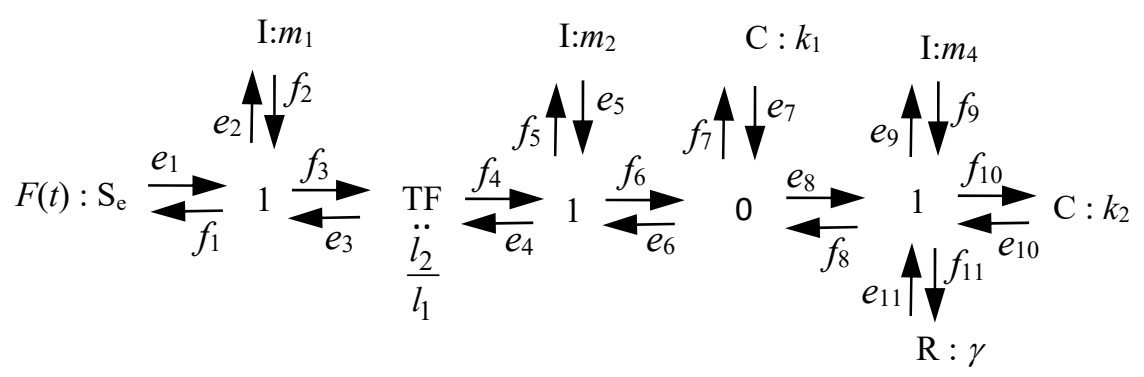

Fig. 3. The signals processing diagram.

The construction of the preliminary block diagram begins with the drawing of two columns corresponding to the node-summation pairs, associated to each junction, as shown in Figure 4. Then, the integrator blocks, the derivative blocks and the gain blocks are represented. In this manner, the preliminary block diagram pictured in Figure 5 is obtained, which, transferred in MATLAB-SIMULINK, leads to the final block diagram (Figure 6). 


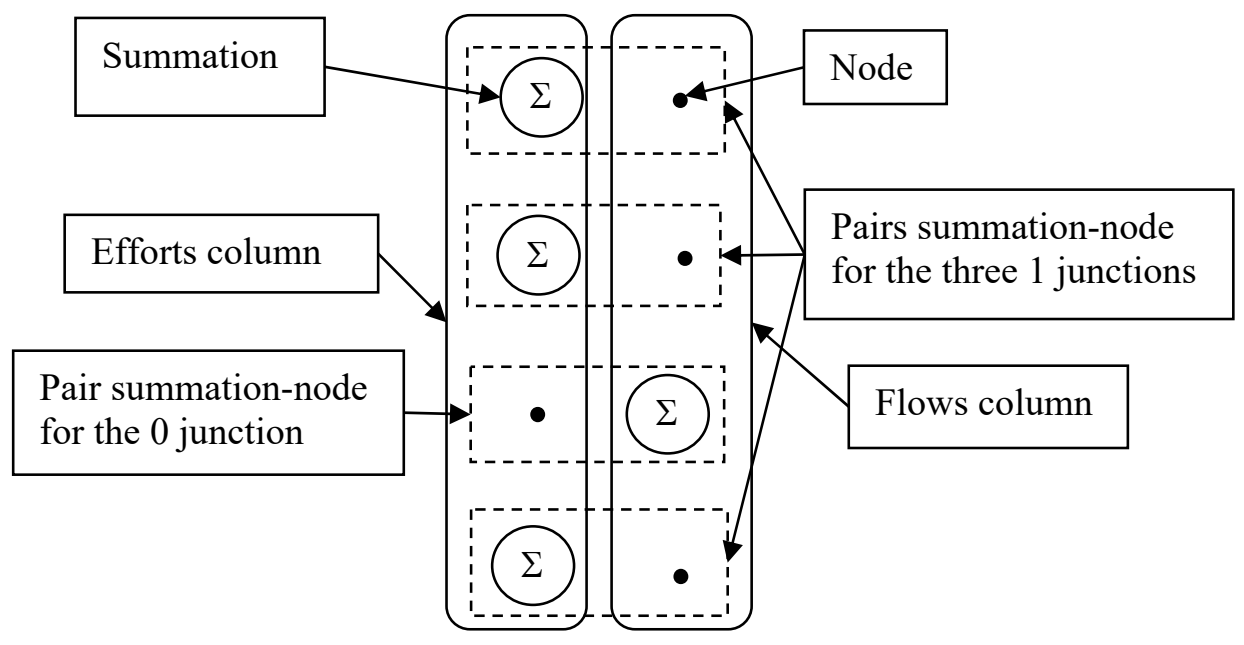

Fig. 4. The junctions representation as summation-node pairs.

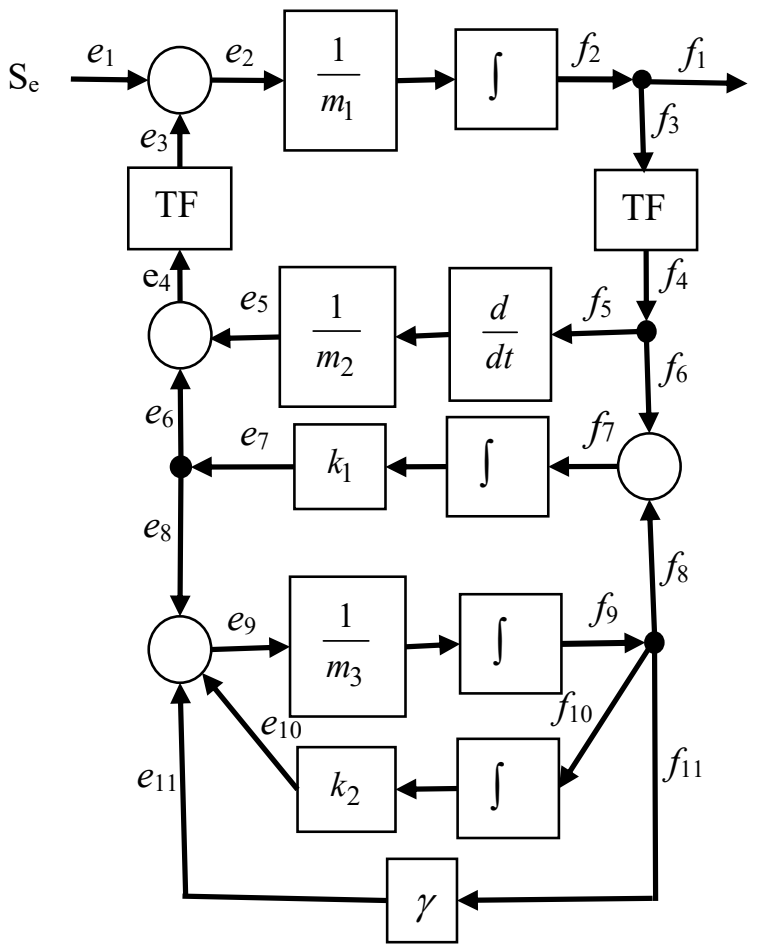

Fig. 5. The preliminary block diagram model. 


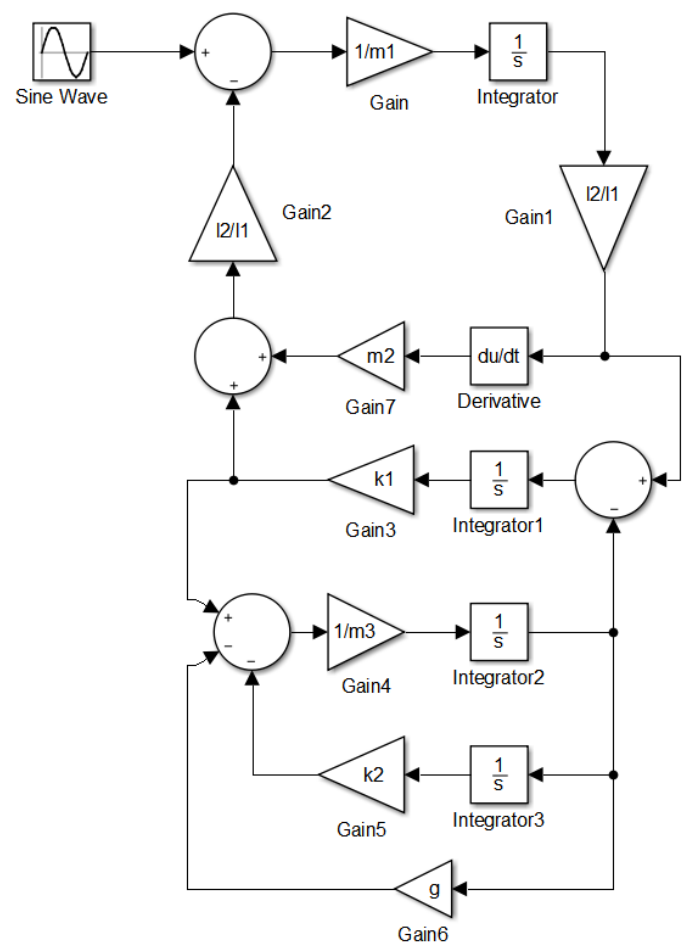

Fig. 6. The block diagram model.

The signs considered in the summation blocks result from the power equilibrium equation written for each junction. For the 1 junction connected to the bonds 1,2 and 3 , the power equilibrium equation resulting from the half arrows direction of the bonds in the bond graph model (Figure 2) is:

$$
e_{1} f_{1}-e_{2} f_{2}-e_{3} f_{3}=0
$$

The input powers have plus sign and the output powers have minus sign. results:

Because the flows on the bonds connected to a 1 junction are equal, that is $f_{1}=f_{2}=f_{3}$, it

$$
e_{1}-e_{2}-e_{3}=0
$$

The output effort from the summation corresponding to this 1 junction is $e_{2}$, as it can be seen in Figure 5. From equation (2), this effort is:

$$
e_{2}=e_{1}-e_{3}
$$

The effort $e_{1}$ will have plus sign and the effort $e_{3}$ will have minus sign in the corresponding summation, as it is shown in the block diagram model (Figure 6).

The procedure is applied to all the summations corresponding to the 1 junctions.

For the 0 junction connected to the bonds 6,7 and 8 , the power equilibrium equation resulting from the half arrows direction of the bonds in the bond graph model (Figure 2) is:

$$
e_{6} f_{6}-e_{7} f_{7}-e_{8} f_{8}=0
$$

The input powers have plus sign and the output powers have minus sign. 
Because the efforts on the bonds connected to a 0 junction are equal, that is $e_{6}=e_{7}=e_{8}$, it results:

$$
f_{6}-f_{7}-f_{8}=0
$$

The output flow from the summation corresponding to this 0 junction is $f_{7}$, as it can be seen in Figure 5. From equation (5), this flow is:

$$
f_{7}=f_{6}-f_{8}
$$

The flow $f_{6}$ will have plus sign and the flow $f_{8}$ will have minus sign in the corresponding summation, as it is shown in the block diagram model (Figure 6).

\section{Conclusions}

It is generally difficult to understand the preliminary block diagram in order to draw it in a dedicated software, due to many crossing signal lines. Because till now there is no a clear methodology for its construction, the present work proposes a systematic method for drawing the preliminary block-diagram by considering two distinct columns, one of them for efforts, the other for flows. In this manner the diagram results very quickly and the signal flow can be easily followed. At the same time, such a block diagram is very easily implemented in MATLAB-SIMULINK, the connection lines being clear, straight and direct.

\section{References}

1. W. Borutzky, Bond Graph Methodology - Development and Analysis of Multidisciplinary Dynamic System Models (Springer-Verlag, London, 2010)

2. R. Ibănescu, O. Păstrăvanu, Bond-Graph Language in Modeling and Simulation of Physical-Technical Systems (in Romanian) ("Gheorghe Asachi” Publishing House, Iași, Romania, 2001)

3. D. Karnopp, D. Margolis, R. Rosenberg, System Dynamics: Modeling, Simulation and Control of Mechatronic Systems (John Willey \& Sons Inc., New Jersey, 2012)

4. R. Ibănescu, Bond Graphs in System Modeling in Graph Based Modelling in Engineering (Springer International Publishing Switzerland, 2017)

5. O. Prakash, A.K. Samantaray, R. Bhattacharyya, Control Engineering Practice, 72,1 (2018)

6. R. Ibanescu, C. Ungureanu, Applied Mechanics and Materials, 809-810, 914 (2015)

7. R. Ibanescu, M. Ibanescu, Materials Science and Engineering, 161, (2016)

8. M.S. Jha, N. Chatti, P. Declerck, Mechanical Systems and Signal Processing, 93, 494 (2017)

9. T.K. Bera, A.K. Samantaray, R. Karmakar, Mechanism and Machine Theory, 49, 2 (2012)

10. S. Lu, Y. Liu, Y. Yao, L. Sun, M. Zhong, Simulation Modelling Practice and Theory, 49, 193 (2014) 